Stanley Fisher1

Fondo Monetario Intemacional y NBER

\title{
La interminable búsqueda de la salvación monetaria
}

\section{Introducción}

El problema monetario ha sido resuelto muchas veces. El problema es encontrar un sistema monetario o una regla que logre la estabilización tanto de los precios agregados como la del nivel del producto alrededor de su tendencia. A lo largo de los siglos ${ }^{2}$, el patrón de oro, la plata, el bimetalismo, el patrón ladrillo, madera, las continuas redefiniciones de la unidad monetaria ${ }^{3}$, una variedad de reglas monetarias incluyendo la doctrina de billetes reales, dinero cien por ciento, reglas de crecimiento monetario constante, niveles de precio o fijación de metas inflacionarias, tipos de cambio fijos, juntas monetarias y otros, todos han sido ofrecidos como la solución al problema. Sin embargo, "la verdad es que el poder adquisitivo del dinero siempre ha sido inestable" (Fisher, 1920, p. XXVI).

Al investigar en la literatura, en el dominio de la palanca monetaria, así como también en muchos de los grandes economistas ${ }^{4}$, cabe preguntarse si estamos buscando la prueba del Último Teorema de Fermat, que después de todo se rindió a la ingeniosidad humana, o en una máquina de movimiento perpetuo. La creencia en los mercados eficientes sugiere esto último. Pero aunque no puede haber una máquina de movimiento perpetuo, algunas máquinas operan más eficientemente que otras, asimismo algunas políticas monetarias son mejores que otras y ha habido progreso en la comprensión de estos temas.

En este trabajo se analizan principalmente los aspectos de un enfoque que hasta hace poco ha recibido mucha atención: la fijación de metas inflacionarias en el contexto de un banco central independiente. Este enfoque no es nuevo, tanto Irving Fisher (1920) como Henry Simons (1948, original en 1935) apoyan la meta del nivel de precios. En este artículo no se realizará un análisis global, pero se tratarán algunos temas clave que respaldan la fijación de metas inflacionarias. 


\section{La independencia del banco central y la fijación de metas inflacionarias ${ }^{5}$}

Debido a que el caso analítico de la independencia del banco central (IBC) parte del sesgo inflacionario que de otra manera estaría presente en la política monetaria, los temas de la IBC y la fijación de metas inflacionarias han estado presentes en la literatura analítica y empírica reciente ${ }^{6}$. Sin embargo, ambos temas son lógicamente distintos: puede favorecerse la independencia del banco central y estar en contra de la fijación de metas inflacionarias o viceversa.

El autor parte del supuesto de que la teoría y la evidencia han demostrado que el banco central debería ser independiente, es decir, que quienes establecen las políticas monetarias no deberían estar bajo el control diario del resto del gobierno $^{7,8}$. Para ser independiente, un banco central necesita tener atribuciones para establecer tasas de interés y - dentro de los límites de la factibilidad técnica- determinar el crecimiento monetario, además de no estar sujeto a tener que financiar al gobierno o a determinados sectores de la economía.

La teoría menciona dos modelos de bancos centrales independientes: el banco central conservador, identificado por Rogoff (1985), y el banco central, que es el esquema de agente principal estudiado por Walsh (1995), Persson y Tabellini (1993). El Bundesbank puede ser tomado como prototipo del modelo de Rogoff, y el Banco de Reserva de Nueva Zelandia como parte de los lineamientos del modelo de agente principal. En la práctica, se observan elementos de ambos modelos en la mayoría de los bancos centrales ${ }^{9}$, los cuales son conservadores; además, sus hábitos sociales fratemales refuerzan sus puntos de vista. En el modelo del banco central conservador, la independencia asegura que las preferencias del banco central prevalecen más que las de la sociedad, en circunstancias en que no es posible un precompromiso en favor de una política de inflación baja. Además, al crear una institución independiente con un liderazgo y gerencia más estable que en los rangos políticos, el sistema hace que sea más probable que las consideraciones de reputación afecten las decisiones de política monetaria, llevando así el resultado inflacionario en la dirección de la solución del compromiso previo.

En el modelo de banco central conservador, se supone que el banco central tratará de moderar el ciclo económico y a la vez luchará contra la inflación. En la práctica, los bancos centrales toman en cuenta la compensación entre la inflación y el producto a corto plazo, decidiendo sobre la velocidad a la cual van a reducir la inflación cuando esté $\multimap$ se espere que esté- por encima de los niveles objetivos, y la velocidad a la cual van a provocar la salida de una recesión. De esta manera satisfacen el supuesto del modelo de Rogoff.

El enfoque de agente principal asigna más énfasis a la definición precisa tanto de la tarea del banco central como de los incentivos que tiene éste para alcanzar sus metas. Al definir claramente metas e incentivos, el enfoque enfatiza 
la responsabilidad del banco central, es decir, la noción de que habrán consecuencias si no alcanza sus metas. Los elementos del enfoque de agente principal se aplican a todos los bancos centrales que tienen diversas metas de política especificadas en la legislación, y cuyos administradores seguramente están motivados tanto por el incentivo de mejorar y mantener su reputación como por las penalidades y recompensas explícitas.

Los dos enfoques enfatizan diferentes elementos de la IBC. Debelle y Fisher (1994) introducen la distinción entre independencia de metas e independencia de instrumentos. Un banco central que tiene el control sobre las palancas de la política monetaria tiene independencia de instrumentos; un banco central que propone sus propias metas de política tiene independencia de metas. El banco central según el modelo de Rogoff tiene ambos tipos de independencia. Por supuesto que el gobiemo trata de escoger al banquero central adecuado, pero - como sucede con los jueces de la Corte Suprema - el comportamiento del banquero central podría ser diferente después de su nombramiento. El banquero central en el modelo de agente principal no tiene independencia de metas, sólo independencia de instrumentos.

La conclusión más importante de la literatura teórica y empírica es que el banco central debería tener independencia de instrumentos, pero no debería tener independencia de metas" conjunto de metas - claramente definida y el poder de alcanzarla, además de asumir la responsabilidad de hacerlo considerando las consecuencias si se fracasa. Esta responsabilidad es necesaria por dos razones: primero, para fijarle incentivos al banco central con el fin de que alcance sus metas y explique sus acciones; $y$, segundo, para proporcionar una supervisión democrática de una institución política poderosa.

Las formas de responsabilidad difieren, como lo podemos ver en el modelo de Nueva Zelandia que responsabiliza al Gobemador y, por consiguiente, al Ministro de Finanzas de una manera precisa; o el modelo de Estados Unidos, que hace responsable a la Reserva Federal aunque no precisamente ante el Congreso; o el modelo alemán, que responsabiliza al Bundesbank ante el público. Dada la importancia de la reputación de los individuos en su vida pública, cada enfoque puede funcionar; sin embargo, la responsabilidad precisa que tienen los funcionarios elegidos es quizá más efectiva que una responsabilidad general y vaga. Además, la responsabilidad de los funcionarios -quienes tienden a llevar vidas muy protegidas - permite a que estén expuestos ante la opinión pública y no se vuelvan demasiado conservadores".

La IBC recae un poco incómodamente en los principios democráticos. Es común hacer analogías con otros organismos independientes dentro del gobiemo general, principalmente con el poder judicial. Sin embargo, los bancos centrales toman decisiones diarias políticamente más importantes que no sólo afectan el 
resultado de las próximas elecciones, sino también porque la adopción de una política monetaria tięne consecuencias destributivas. La responsabilidad, que hasta cierto punto limita la independencia del banco central, ayuda a mantener un equilibrio apropiado entre los poderes del banco central y el gobierno.

El apoyo intelectual más fuerte para la IBC proviene de los resultados empíricos que muestran que, entre los países industrializados, el resultado inflacionario promedio guarda una relación negativa con el grado de independencia legal del banco central (Alesina y Summers, 1993). Se ha dicho que la relación no es causal $^{12}$. En cambio, los países que son en efecto aversos a la inflación, debido por ejemplo a su historia hiperinflacionaria, desarrollan instituciones que respaldan esta aversión. Este argumento sugeriría que la educación del público con respecto al verdadero costo de la inflación sería la mejor manera para reducirla. Sea como sea, a menos que las leyes fueran totalmente irrelevantes con respecto al desempeño, cualquiera que quiera reducir la inflación debería respaldar activamente la causa de la IBC. El mayor apoyo a la IBC ha estado acompañado por la tendencia de adjudicarle al banco central la tarea -única- de alcanzar una tasa o nivel objetivo de inflación ${ }^{13}$. Típicamente, la meta inflacionaria es de un nivel de inflación para el próximo año o dos, o si no de una trayectoria inflacionaria (también dentro de un rango) a lo largo de varios años. La elección de una meta inflacionaria plantea varios aspectos: si sería mejor escoger una meta que fuera directamente más controlable por el banco central, tal como el crecimiento de un agregado monetario en sentido estricto; si la inflación debiera ser el único objetivo, dado que la política monetaria afecta tanto el producto como la inflación a corto plazo, y si es preferible especificar una meta de ingreso nominal en lugar de una meta inflacionaria; si escoger un nivel de precios en vez de un objetivo de tasa de inflación; a qué nivel y sobre qué horizonte especificar el objetivo y cómo cambiarlo, si es que debe cambiarse; y si mejor escogemos un tipo de cambio vinculado en lugar de una meta inflacionaria.

Obviamente sería mejor que el banco central fijara una variable de política directamente bajo su dominio que también controla muy de cerca una variable objetivo, tal como la tasa de inflación o el producto nominal. Durante algún tiempo se tuvo la esperanza de que la fijación de metas monetarias lograría este propósito, pero a medida que la relación entre el crecimiento monetario y la inflación y/o el producto se ha ido destruyendo en un país después del otro, no ha sido posible para ningún país depender solamente de la fijación de metas inflacionarias $^{14}$.

La cuestión de si el banco central debería perseguir una meta que esté directamente bajo su control en vez de un objetivo eventual de política, como la tasa de inflación, toca un aspecto clave de los enfoques recientes sobre la IBC. Muchas de las investigaciones sobre política monetaria realizadas en las últimas cuatro décadas han tratado de descubrir una regla monetaria óptima, ya sea una 
tasa de crecimiento constante para alguna medida de dinero definida en sentido estricto; una regla de retroalimentación, tal vez para la base monetaria, como en MacCallumm (1994), o una regla de tasa de interés. Friedman (1960, p. 90) propuso que el Congreso debería encomendar a la Reserva Federal que siguiera la regla de la tasa de crecimiento constante. En cambio, en la literatura reciente se deja implícita y apropiadamente en manos del banco central la decisión de qué política monetaria adoptar para alcanzar las metas: una razón para tener un banco central es centralizar tanto la capacidad como la responsabilidad de hallar la mejor forma de implementar la política monetaria ${ }^{15}$.

Suponiendo que un banco central independiente tenga acceso a todos los conocimientos relevantes acerca de cómo trazar la política monetaria para lograr sus objetivos, ¿por qué darle una meta inflacionaria en lugar de metas de inflación y producto, o el objetivo de maximizar la función de utilidad para la sociedad? Una respuesta ilegítima es que la inflación es un fenómeno monetario y, por tal razón, la política monetaria debería de estar enfocada a la inflación. Este enunciado es corecto, pero evade más que abordar el hecho de que siempre hay una compensación a corto plazo entre la inflación y el producto, y que la elección de la política monetaria afecta el producto a corto plazo así como también a la inflación.

Fundamentalmente, tanto el enfoque del banco central conservador como el de agente principal, ya sea directa o de manera implícita, suponen que el banco central perseguirá ambos objetivos ${ }^{16}$. En el enfoque de agente principal, la tasa de inflación objetivo se ve afectada por el estado de la economía, lo que implica por ejemplo que los shocks de oferta afecten la tasa de inflación objetivo ${ }^{17}$. En el caso de Nueva Zelandia, la meta inflacionaria se ajusta automáticamente por cambios en los términos de intercambio y por los impuestos indirectos; esto quiere decir que la inflación que ocurre como resultado de estos shocks se acomoda, para poder reducir su efecto sobre el producto.

El argumento más poderoso para la fijación de metas inflacionarias, con ajustes para shocks en lugar de fijar objetivos para dos variables, es que la responsabilidad se incrementa cuando el banco central recibe un objetivo único en vez de múltiples objetivos. Sin embargo, las metas en materia de producto y de inflación se pueden combinar en un indicador único u objetivo, siendo el PIB nominal un indicador igual que cualquier otro ${ }^{18}$. Hay dos dificultades principales con el objetivo del ingreso nominal: primero, y más fundamentalmente, los datos sobre el PIB nominal aparecen con retraso y se revisan con frecuencia; y segundo, esta información parece ser de poco interés directo para el público. El problema de la revisión de los datos siempre es severo.

El argumento en favor de la fijación de metas inflacionarias en vez de una meta de ingreso nominal, es que la tasa de inflación preocupa directamente a los agentes económicos y que el comportamiento de la inflación es más fácil de vigilar que el del ingreso nominal. Las metas inflacionarias proporcionan la res- 
puesta adecuada de la política monetaria ante los shocks de demanda, es decir, que la política monetaria debería hacerse más restrictiva en respuesta a los shocks que tenderían a aumentar tanto el producto como la inflación. Como un shock de ofer $\_$lleva a precios más altos y menor producto, la política monetaria tenderá a ser menos restrictiva en respuesta a un shock adverso de oferta en el caso de fijarse un objetivo de ingreso nominal que en el caso de un objetivo inflacionario. Entonces, el objetivo de ingreso nominal tiende a implicar una respuesta más automática de la política monetaria frente a los shocks de oferta. Esta ventaja se ve contrarrestada en la medida en que la fijación de metas inflacionarias contenga una provisión especial para los shocks de oferta, como sucede de diferentes maneras en Gran Bretaña, Canadá y Nueva Zelandia. El autor supone que la fijación de una meta inflacionaria es preferible al objetivo de ingreso nominal, siempre que la meta se ajuste para los shocks de oferta. ¿Y qué sucede con la opción entre la tasa de inflación objetivo y la trayectoria objetivo del nivel de precios? Cuando el objetivo de una política es la trayectoria del nivel de precios, tiene que contrarrestar los shocks inflacionarios pasados con un período de inflación inferior del promedio para poder regresar a la trayectoria objetivo. Como se demuestra en Fisher (1994), la fijación de metas inflacionarias tiende a producir más certeza con respecto al nivel de precios en el futuro próximo, a costa de una mayor incertidumbre sobre el nivel de precios en un futuro distante. De manera equivalente, la tasa de inflación fluctuaría más a corto plazo con un objetivo de nivel de precios, a medida que la política procura regresar a la trayectoria de precios escogida. Por ejemplo, con el objetivo del nivel de precio, el Bundesbank, hoy en día, al acercarse a una tasa de inflación del 2 por ciento, tendría que reducir la inflación por debajo del 2 por ciento durante todo el tiempo que fuera necesario para neutralizar los efectos de la inflación superior al promedio del período desde 1990. Si la meta es estimular la contratación nominal a largo plazo, entonces el objetivo del nivel de precios sería preferible. Sin embargo, ya que la mayoría de contratos nominales son a corto plazo, y puesto que la función de la política monetaria sería mucho más exigente con un objetivo de nivel de precios y los beneficios de la contratación nominal a largo plazo podrían lograrse igualmente mediante la indización, la meta inflacionaria es preferible al objetivo del nivel de precios.

Las metas inflacionarias se especifican de dos maneras. La primera es fijar una tasa de inflación objetivo a largo plazo, pero no una trayectoria para la tasa. Por ejemplo, el Bundesbank tiene una meta de inflación base de cerca del 2 por ciento, pero no especifica la trayectoria por medio de la cual se propone regresar al objetivo cuando la inflación exceda el 2 por ciento. Los bancos centrales con objetivos de inflación más formales típicamente especifican un nivel de inflación para los próximos años, que puede modificarse en forma periódica. Se presume que la meta sólo será creible si dicho nivel es consistente con otras políticas que quizá continúe el gobiemo. Si bien la necesidad de credibilidad 
limita las metas que puede establecer el banco central, éste tiene también que reconocer que sus propias decisiones quizá afecten tanto el comportamiento del gobierno como el del sector privado.

\section{Vinculación del tipo de cambio}

El análisis de la fijación de metas inflacionarias sería relevante incluso en un sistema de tipo de cambio fijo, en el cual debe especificarse la base de la política monetaria para el mundo o el país en donde la moneda se utilizará como ancla. La cuestión del retorno a un sistema de cambio fijo, o uno con niveles objetivo entre las principales monedas, continúa en discusión por investigadores como Williamson (1994). Aunque los acontecimientos pueden cambiar con rapidez las opiniones sobre los regímenes de tipo de cambio, cualquier movimiento hacia zonas objetivos entre el yen, el dólar, y el marco alemán, o la meta más ambiciosa de una moneda mundial, ciertamente está muy lejos, a pesar de la posibilidad cada vez mayor de que algunos países europeos formen una unión monetaria antes de comenzar el próximo siglo.

Muchos países deben optar entre tipos fijos y flotantes, así como entre una variedad de regímenes intermedios, como un peón gateador o un vínculo móvil o un vínculo móvil con bandas. Puesta a un lado la credibilidad, y para una política fiscal específica, la opción entre tasas fijas y flotantes depende de la flexibilidad de los precios y de la fuente predominante de shocks que tiene que enfrentar la economía. Una economía que tal vez enfrente shocks nominales preferirá un tipo de cambio fijo, permitiendo que la oferta monetaria se adapte a las perturbaciones. Una economía que enfrente significativos shocks reales, incluyendo cambios en la afluencia de capital a corto y largo plazo, se beneficiará permitiendo que el tipo de cambio aborde parte del peso del ajuste y, en consecuencia, tendrá un tipo flexible. De manera similar, si la política fiscal no es sostenible y es inestable, entonces el tipo de cambio fijo es menos persuasivo ${ }^{19}$.

El régimen cambiario puede ser transitorio. Por ejemplo, las economías con alta inflación pueden utilizar un tipo fijo temporal como ancla nominal clave en un programa de desinflación coordinada. Siempre que las variables fundamentales sean correctas (especialmente que la política fiscal sea consistente con una tasa de inflación baja y que el tipo de cambio no esté sobrevaluado) ${ }^{21}$, una vinculación del tipo de cambio podría ayudar a reducir la inflación con más rapidez que un régimen de tipo de cambio flotante, en el cual las variables fundamentales de la economía deben ajustarse al sistema de salarios-precios. Dependiendo del proceso de determinación de salarios en el país, también podría ser posible coordinar una disminución en la inflación de los salarios con el programa de estabilización. Debido a que en dichos programas la inflación no llega a 0 de inmediato, el tipo de cambio real quizá se aprecie al comienzo del programa; el valor inicial del tipo de cambio debe tomar en cuenta esta apreciación. 
Es difícil decir en abstracto si la vinculación del tipo de cambio agrega credibilidad a la orientación antinflacionaria de la política monetaria. Esta vinculación puede ser más o menos creíble, dependiendo de las políticas del país y de su habilidad y deseo de defender el vínculo ${ }^{21}$. El tipo de cambio fijo probablemente fortalecerá la mano del banco central y le dará una razón adicional para resistir la presión de la autoridad fiscal, y también podría concentrar las mentes del banco central y del gobiemo en la meta de preservar el tipo de cambio, y así agregarle coherencia a la política. Esta claro, sin embargo, que existen pocos palses en los que las fuerzas políticas no cuestionarán de manera eventual un vínculo que esté causando dificultades severas. Suecia, en 1993, lo defendió casi a muerte, pero las tasas de interés del 500 por ciento no eran creibles. Aun dentro del SME en 1993, donde sin duda varios gobiernos querían evitar la devaluación, el sistema existente fue abandonado. Debido a que ningún arreglo de tipo fijo ha persistido para siempre en ausencia de una moneda común, y debido a que los riesgos financieros de los participantes en el mercado son altos, el mercado siempre estará en guardia frente a la posibilidad de devaluación.

No obstante, cuando la política monetaria ha perdido credibilidad, y cuando el banco central tiene que establecer una nueva política, un régimen de tipo de cambio fijo puede ser la forma más clara para que el banco central indique su seriedad y logre o recobre la credibilidad. No hay otra política que pueda ser tan bien vigilada por el público, especialmente si el banco central publica en forma oportuna su información monetaria y de reserva. En el caso extremo, esto tomaría la forma del establecimiento de una junta monetaria, como sucedió en el caso de Argentina, Hong Kong (ambos vinculados al dólar) y Estonia (al marco alemán) $)^{22}$. En el enfoque de la junta monetaria, el tipo de cambio se fija y la base monetaria está completamente cubierta por reservas de moneda extrajera ${ }^{23}$.

Las metas inflacionarias no se excluyen en un régimen de vínculo fijo o móvil. En programas de estabilización, el vínculo fijo o móvil puede ser una parte crucial de un programa coordinado antiinflacionario. Sin embargo, debe reconocerse que ante los shocks - como una afluencia inesperada de capital- los dos objetivos pueden resultar inconsistentes si son especificados en forma rígida. Esa es una razón por la cual es aconsejable, en algunos casos, establecer una banda alrededor del tipo de cambio si la meta inflacionaria es el objetivo primordial de la política.

En varios episodios bien conocidos al gobiemo le ha resultado difícil salir de un arreglo de tipo de cambio fijo sin una crisis. El episodio reciente más obvio es el de México. La economía política de deshacerse de un vínculo es complicada, y cuanto más tiempo se quede el gobiemo con este programa inapropiado, más costoso le resultará devaluar. No hay una regla precisa para saber cuándo debe abandonarse un vínculo o cuándo hacerlo más flexible, pero tiene que hacerse una vez que esté claro que el tipo de cambio está significativamente 
sobrevaluado, y la mejor indicación de la sobrevaluación es la situación real o prevista de la cuenta corriente ${ }^{24}$. No tenemos todavía indicadores sencillos de la sostenibilidad de los déficit ${ }^{25}$, pero la teoría que se ha utilizado para examinar la sostenibilidad de los déficit fiscales ${ }^{2 h}$ puede transferirse a la sostenibilidad del déficit en cuenta corriente, con la advertencia de que toda una gama de factores adicionales, incluyendo la estructura de vencimientos de la deuda y la posibilidad de cambios en la actitud del mercado, necesitan ser tomados en cuenta e implican bandas de confianza muy amplias alrededor de la estimación central. A pesar de la dificultad de encontrar un criterio preciso para determinar el momento adecuado para abandonar un vínculo, muchos países, entre ellos Israel, en 1986, y Polonia, en 1990, han logrado alejarse de un tipo fijo transitorio sin una crisis grave.

\section{Conclusión}

El enfoque de fijar metas inflacionarias en el contexto de un banco central independiente pero responsable, es un aspecto nuevo y significativo en la búsqueda interminable de la salvación monetaria. El comportamiento inflacionario de los tres países que han estado aplicando metas inflacionarias - Canadá, Nueva Zelandia y el Reino Unido- ha sido favorable en los últimos años. Sin embargo, para juzgar el éxito de este enfoque debemos esperar probarlo en las circunstancias difíciles de una economía recalentada ${ }^{27}$.

Está claro, además, que la política monetaria no puede asegurar un buen comportamiento macroeconómico, a menos que la política fiscal opere de manera razonable. Mas (1994) argumenta que la IBC no es la clave para la estabilidad monetaria; la clave es asegurar la disciplina fiscal. El argumento podría expresarse como que una enmienda de presupuesto equilibrado es más útil para preservar el valor del dinero que el establecimiento de un banco central independiente ${ }^{28}$. Como es bien conocido, las evidencias empíricas demuestran que, para los países en desarrollo, las medidas de la extensión de la independencia legal del banco central están relacionadas positivamente con la inflación, resultado que en parte podría reflejar el problema fiscal al cual se refiere Mas.

Utilizar un vínculo cambiario proporcionando un ancla nominal visible, también puede desempeñar un papel muy importante en la reducción de la inflación y las expectativas inflacionarias. Este es, en particular, el caso de una economía que busca estabilizarse después de una situación de extremo desorden y en la que la credibilidad del banco central es baja. Para ser efectiva, dicha estrategia debe partir de un tipo de cambio apropiado y estar acompañada de variables fundamentales sólidas (especialmente en materia fiscal) y de la disposición del gobierno de modificar el tipo de cambio cuando sea necesario.

Si bien los arreglos monetarios adecuados ciertamente pueden ayudar a lograr estabilidad en los precios, cada arreglo será puesto a prueba en cierto mo- 
mento, pero aún no existen arreglos que permitan mantener la inflación baja a un menor costo en todas las circunstancias.

24 de junio de 1999.

\section{Referencias bibliográficas}

Alestina, A y Summers, L.H. "Central bank independence and macroeconomic performance: Some comparative evidence". Journal of Money, Credit and Banking, 25, vol. 2, 1993, pp. 151-162.

Bennett, A.G.G. "Currency boards: Issues and experiences". IMF. Paper on Policy Analysis and Assessment, septiembre, 1994.

Calvo, G. Varieties of capital market crises. Universidad de Maryland. Documento de Trabajo, 1995.

Calvo, G.; Leiderman, L. Y Reinhart, C. The capital inflows problem. IMF. Paper on Policy Analysis and Assessment, julio, 1993.

Clapham, J. The Banck of England, vol. II. Nueva York: Macmillan, 1945.

Cukierman, A. Central bank strategy, credibility, and independence. Cambridge, MA.: MIT Press, 1992.

Debelle, G. y Fischer, S. "How independent should a central bank be?". En Goals, guidelines, and constraints facing monetary policymakers, J. Fuhrer (ed.). Banco de Reserva Federal de Boston. Serie de conferencias, No. 38, 1994.

Eijffinger, S. y de Haan, J. "The political economy of central bank independence". Centro para la investigación económica. Tilburg University, the Netherlands. Mimeo, 1995.

Fischer, S. "Modern central banking". En The future of central banking, Capie, F.; Goodhart, C.; Fischer, S.; y Schadt N (eds.). Cambridge University Press, 1994, pp. 262-308.

Fischer, S. "Central bank independence in the transition economies". IMF. Mimeo. Banco Nacional de Hungría, 1995a.

Fischer, S. Central-bank independence revisited. American Economic Review, Papers and Proceedings, 85, vol. 2, 1995b, pp. 201-206.

Fisher, I. Stabilizing the dollar. Nueva York: MacMillan, 1920.

Friedman, M. A program for monetary stability. Nueva York: Fordham University Press, 1960.

Hall, R.E. y Mankiw, N. "Nominal income targeting”. En Monetary policy, N.G. Mankiw (ed.). Chicago: University of Chicago Press, 1994, pp. 71-93.

Krugman, P. "A model of balance-of-payments crises". Journal of Money, Credit, and Banking, 11, vol. 3, 1979, pp. 311-325.

Mas, I. Central bank independence: A critical view. The World Bank. Policy Research Working Paper 1356, septiembre, 1994.

McCallum, B.T. Monetary policy rules and financial stability. NBER Working Paper 4692, septiembre, 1994.

Nordhaus, W.D. "Policy games: Coordination and independence in monetary and fiscal policies". Brookings Papers on Economic Activity, 2, 1994, pp. 139-199.

Persson, T. Y Tabellini, G. "Designing institutions for monetary stability". Carnegie Rochester Conference Series on Public Policy, 39, diciembre, 1993, pp. 53-84. 
Posen, A. Declarations are not enough: Financial-sector sources of central bank independence, 1995.

Rogoff, K. "The optimal degree of commitment to an intermediate monetary target". Quarterly Journal of Economics, 100, vol. 4, 1985, pp. 1169-1190.

Schnadt, N. y Whittaker, J. "Is indirect convertibility impossible?". Journal of Money, Credit and Banking, 27, vol. 1, 1995, pp. 297-298.

Simons, H.C. "Rules versus authorities in monetary policy". En Economic policy for a free society, H.C. Simons (ed.). Chicago: University of Chicago Press, 1948, pp. 160183.

Svensson, L.E.O. "Fixed exchange rates as a means to price stability: What have we learned?". European Economic Review, 38, 1994, pp. 447-468.

Svensson, L.E.O. The Swedish experience of an inflation target. NBER Working Paper No. 4985 , enero, $1995 a$.

Svensson, L.E.O. Inflation targeting need not increase output variability: Comment on Rogoff and Walsh. Institute for International Studies, University of Estocolmo, abril, $1995 b$.

Walsh, C. "Optimal contratcs for central bankers". American Economic Review, 85, vol. 1, 1995, pp. 150-167.

Wilcox, D.W. "The sustainability of government deficits: Implications of the presentevalue borrowing constraint". Journal of Money, Credit and Banking, 21, vol. 3, 1989, pp. 291-306.

Williamson, J. (ed.). Estimating equilibrium exchange rates. Washington: Institute for International Economics.

\section{Notas}

1. Con licencia del MIT. Este ensayo fue preparado para la Conferencia sobre Macroeconomía de la NBER, Cambridge, Massachusetts, realizada del 10 al 11 de marzo de 1995. Los puntos de vista expresados son propiamente del autor y no deberán atribuirse al Fondo Monetario Internacional.

2. Los escritores anteriores se concentraron más en el comportamiento de los precios y los pánicos financieros que en la estabilización del producto.

3. Este enfoque se relaciona con el concepto conocido como la convertibilidad indirecta; ver Schnadt y Whittaker (1995).

4. Al escribir acerca de las controversias monetarias hace un siglo, Clapham (1945, vol. 2, p. 313) comenta: "Pero la caída del precio [del nivel de precios agregados] y los problemas de la plata reviven el interés de la cuestión, de manera que por cerca de 20 años — desde finales de los años setenta hasta finales de los noventa- ejercitó y atrajo a mentes agudas, y nubló ese tipo tan familiar de mente más sencilla que espera perennemente que suscedan todas las cosas buenas a través de la manipulación de la moneda".

5. Esta sección se basa en Fisher (1994), que incluye una investigación completa de los enfoques recientes sobre el tema de la independencia del banco central y la fijación de metas inflacionarias, y Fisher (1995b); ver también Debelle y Fisher (1994).

6. Gran parte de la investigación anterior se presenta en Cukierman (1992). Para una investigación reciente y global, ver Eijffinger y de Haan (1995). 
7. Si bien el banco central es parte del gobierno de un país - de ahí el dicho de que "el banco central debería ser independiente dentro del gobierno, pero no del gobierno"-, por conveniencia me referiré al "gobierno" como algo que excluye al banco central.

8. Esto significa que supongo que los beneficios de la política monetaria independiente sobrepasan — no evitan - los beneficios de la coordinación de política que han sido enfatizados por Nordhaus (1994).

9. Svensson (1995b) muestra que los dos enfoques pueden producir un comportamiento equivalente si el banco central de Rogoff tiene un objetivo inflacionario más bajo que el de la sociedad.

10. La base de estas conclusiones se expresa en Fisher (1994).

11. La posibilidad de que el banco central sea demasiado independiente, e n el sentido de que sus políticas no tengan suficientemente en cuenta los movimientos del producto, se analiza en Debelle y Fisher (1994). Si bien la responsabilidad de los funcionarios elegidos es deseable dentro de un sistema que funciona en democracia, la cuestión es más complicada en otros casos. En Fisher (1995a), el autor sostiene que durante el período de transición, los bancos centrales deberían, en algunas de las economías de transición en las que existe la posibilidad de populismo parlamentario, tencr independencia sin responsabilidad de los funcionarios elegidos. La responsabilidad sería, cntonces, implícitamente ante la opinión pública o la historia, como el caso del Bundesbank.

12. Posen (1995) sostiene que el grado de aversión inflacionaria cs determinado, en gran medida, por los puntos de vista del sector financiero, existicndo países con sectores financieros más opucstos a la inflación que tienen bancos centrales más independientes.

13. Ciertamente, algunos invetigadores miden la IBC por la medida en la cual el banco central tiene una única meta de estabilidad de precios o baja inflación (Cukierman, 1992, capítulo 19).

14. No hay nada más común después de que parece habersc quebrantado una función de demanda monetaria, que la demostración de que la demanda de alguna definición de dinero era estable. Esos ejercicios ex post no son muy útiles.

15. Esto no quiere decir que los cconomistas no deban continuar investigando estos tcmas; solamente que no debcrían esperar ver sus resultados consagrados en la legislación.

16. Como se explicó anteriormente, se supone que el banco central conscrvador, de acuerdo con el enfoque de Rogoff, toma en cuenta las metas de producto y de inflación.

17. Esto puede verse claramente en la formulación de Persson y Tabcllini (1993).

18. Hall y Mankiw (1994) sostienen que el objetivo del ingreso nominal sería una buena regla monetaria, pero que podría mejorarse en las formas que se especifiquen.

19. Sin embargo, podría ser que la adopción de un régimen de tipo de cambio fijo contribuya a restringir la política fiscal.

20. En economías en transición, una guía útil para el tipo de cambio nominal apropiado es el valor en dólares de los salarios.

21. Svenssson (1994), escribiendo despućs de las crisis cambiarias de 1993 en el MAC y en los países nórdicos, argumenta quc: "los tipos de cambio fijos no son un atajo 
a la estabilidad de precios. La estabilidad monetaria y la credibilidad tienen que ser logradas en casa" (p. 447). Si bien la credibilidad realmente tiene que ser establecida a través de la política interna, no veo por qué un vínculo cambiario no contribuiría a lograr la estabilidad de los precios.

22. Bennett (1994) describe estas tres juntas mentarias y presenta una evaluación muy útil de la política monetaria.

23. En el sistema de junta monetaria más estricto no hay banco central y el volumen de la base monetaria está determinado puramente por la balanza de pagos. Este también es el caso en el mecanismo de flujo de Hume que proporciona la teoría más simple de la operación automática del patrón oro: un superávit en cuenta corrriente conduce a una expansión de la oferta monetaria, y un déficit conduce a una reducción de la of erta monetaria; los movimientos del volumen de dinero equilibran la cuenta corriente. La situación es más complicada cuando la cuenta de capital tiene que ser tomada en cuenta.

24. También está la regla general: "Se sabe que el tipo de cambio está sobrevaluado cuando el Ministro de Finanzas le dice que dirija su atención a un índice de precios muy obscuro de acuerdo al cual el tipo de cambio real no está sobrevaluado".

25. Williamson (1994) habla de este asunto.

26. Ver, por ejemplo, Wilcox (1989). El análisis también debería incluir la posibilidad de un ataque sobre las reservas, como en el modelo básico de Krugman (1979). Ver Calvo (1995) para un análisis de estos temas.

27. Svensson (1995a) analiza la experiencia sueca en este sentido, que ha sido menos exitosa y menos creible.

28. Esta proposición no la acepta el autor. 